\title{
Las actividades lúdicas y el estado emocional en estudiantes de educación básica superior
}

\section{Play activities and emotional state in students of higher basic education}

1 Diego Javier Mayorga Ortiz

https://orcid.org/0000-0001-8201-3582

Universidad Técnica de Ambato, Facultad de Ciencias de la Educación, Carrera de

Pedagogía de la Actividad Física y Deporte. Tungurahua, Ecuador,

dj.mayorga@uta.edu.ec

2 Christian Mauricio Sánchez Cañizares (iD) https://orcid.org/0000-0002-0677-7246

Universidad Técnica de Ambato, Facultad de Ciencias de la Educación, Carrera de

Pedagogía de la Actividad Física y Deporte. Tungurahua, Ecuador,

cm.sanchezc@uta.edu.ec

3 William Rubén Mayorga Ortiz (iD) https://orcid.org/0000-0003-3318-3061 Universidad Técnica de Ambato, Facultad de Ciencias de la salud, Docente de posgrado de la especialidad de Medicina Familiar y Comunitaria. Tungurahua, Ecuador, wmayorga9465@uta.edu.ec

4 Kleber Washington Morales Carpio (iD) https://orcid.org/0000-0003-2903-0185 Universidad Técnica de Ambato, Facultad de Ciencias de la Educación, Carrera de Pedagogía de la Actividad Física y Deporte. Tungurahua, Ecuador, kmorales7243@uta.edu.ec

Artículo de Investigación Científica y Tecnológica Enviado: 24/12/2021

Revisado: 29/12/2021

Aceptado: $12 / 01 / 2022$

Publicado:08/03/2023

DOI: https://doi.org/10.33262/concienciadigital.v6i1.4.2030

Cítese: Mayorga Ortiz, D. J., Sánchez Cañizares, C. M., Mayorga Ortiz, W. R., \& Morales Carpio, K. W. (2023). Las actividades lúdicas y el estado emocional en estudiantes de educación $\begin{array}{llll}\text { básica } & \text { superior. } & \text { ConcienciaDigital, } & \text { 812-825. }\end{array}$ https://doi.org/10.33262/concienciadigital.v6i1.4.2030

\footnotetext{
CONCIENCIA DIGITAL, es una Revista Multidisciplinar, Trimestral, que se publicará en soporte electrónico tiene como misión contribuir a la formación de profesionales competentes con visión humanística y crítica que sean capaces de exponer sus resultados investigativos y científicos en la misma medida que se promueva mediante su intervención cambios positivos en la sociedad. https://concienciadigital.org

La revista es editada por la Editorial Ciencia Digital (Editorial de prestigio registrada en la Cámara Ecuatoriana de Libro con No de Afiliación 663) www.celibro.org.ec 


\section{Palabras} claves: actividades lúdicas, estados emocionales, educación física.
Keywords: recreational activities, emotional states, physical education.
Resumen

Dentro de la actividad física una herramienta eficaz en la pedagogía es el uso de actividades lúdicas, siendo una estrategia didáctica que genera grandes beneficios al desarrollo de la asignatura de cultura física; incidiendo en tres aspectos fundamentales en los estudiantes como son: las capacidades físicas, sociales y psicológicas. Objetivo: En la presente investigación se analizó la asociación de las variables actividades lúdicas y el estado emocional de los estudiantes. Metodología: Se utilizó un enfoque cuantitativo, con una finalidad básica de diseño no experimental, con un alcance descriptivo con un método del nivel teórico hipotético-deductivo. En la recolección de datos se utilizó el DASS 21 y la encuesta para evaluar cada variable en una muestra de estudio de 59 estudiantes.

Resultados: Fueron determinados mediante la prueba estadística chi cuadrado con diferenciación asintótica bilateral en un nivel de $\mathrm{P} \leq 0.05$ dando como consecuencia un valor estadístico significativo en el nivel de asociación del uso de actividades lúdicas y el estado emocional de los estudiantes. Conclusión: Se concluye que el uso de actividades lúdicas actúa como factor protector para evitar que se desarrollen estados de ánimo negativos en los estudiantes.

\section{Abstract}

Within physical activity, an effective tool in pedagogy is the use of recreational activities, being a didactic strategy that generates great benefits for the development of the physical culture subject; focusing on three fundamental aspects in students such as: physical, social, and psychological capacities. Objective: In the present investigation the association of the variable's recreational activities and the emotional state of the students is analyzed. Methodology: A quantitative approach was used, with a basic purpose of nonexperimental design, with a descriptive scope with a method of the hypothetical-deductive theoretical level. In the data collection, the DASS 21 and the survey were used to evaluate each variable in a study sample of 59 students. Results: They were determined using the chi-square statistical test with bilateral asymptotic differentiation at a level of $\mathrm{P} \leq 0.05$, resulting in a significant statistical value in the level of association between the use of recreational activities and the emotional state of the students. Conclusion: It is concluded that the use of playful activities acts as 
a protective factor to prevent negative moods from developing in students.

\section{Introducción}

Dentro de la actividad física una herramienta eficaz en la pedagogía es el uso de actividades lúdicas, siendo una estrategia didáctica que genera grandes beneficios a las clases de cultura física; incidiendo en tres aspectos fundamentales en los estudiantes como las capacidades físicas, social y psicológicas, un aspecto poco estudiado es la incidencia que tiene las actividades lúdicas en los estados emocional de los educandos, Por lo cual se analizara la asociación que existe entre la actividad lúdica y el estado emocional de los estudiantes.

La actividad lúdica es un proceso didáctico que se realiza mediante el uso o no de un instrumento externo o interno el cual presenta un objetivo determinado Bravo et al. (2021). La cual fomenta una dimensión del desarrollo humano que busca el conocimiento, la formación de la autoestima y del desarrollo psicosocial de los educandos donde existe un grado de diversión (Palacios y Jiménez, 2002). Se conceptualiza como una actividad de carácter infantil presente en las etapas de desarrollo el cual influye en su desarrollo afectivo e intelectual, el juego es un medio de aprendizaje autónomo y natural que se lo desarrolla en los tiempos libres o en las aulas de clase (Benitez y Gamarro, 2010). En el ámbito educativo el uso del juego como una herramienta para el proceso de enseñanza aprendizaje se convierte en actividades lúdicas que son estrategias pedagógicas que permiten generar conocimiento, además el juego presenta un carácter lúdico, siendo una herramienta esencial para el docente en el desarrollo socio educativo en función de las necesidades del niño y el adolescente (Mocha y Bonilla., 2018). La metodología lúdica busca interiorizar en los estudiantes propiciando el autoconocimiento propio del juego y transformarlo en tiempo significativo para el trabajo académico (Romero et al., 2009). En las clases de cultura física una estrategia didáctica a utilizar es la actividad lúdica que es de carácter física en donde todos los órganos del cuerpo se activan, además de ayuda a que se fortifiquen y que se ejerciten otras funciones del cuerpo, por ejemplo, las funciones cognitivas (Gómez et al., 2017).

El juego es un arma poderosa para los docentes en su búsqueda de lograr un aprendizaje holístico en donde el niño no solo aprende a saltar o a practicar un deporte sino que también se prepara para poder tener una vida social, aprender valores como el respeto y la solidaridad al realizar juegos cooperativos, ayuda en la formación de su carácter mediante los juegos competitivos que conllevan una victoria o una pérdida y estimula a su creatividad por la libertad que tienen los juegos, en cuanto a su formación individual, los juegos en las clases de cultura física despiertan el ingenio y el desarrollo de muchas 
habilidades físicas, favorecen en su capacidad visual, táctil y auditivas, les brindan soltura, confianza sobre su aspecto físico y emocional (Osorio et. al., 2013).

Los resultados que se obtiene al utilizar actividades lúdicas en el contexto educativo se observan en tres niveles que son el biológica, psicológica y social, por ello podemos afirmar que el juego en la educación es trascendente y vital (Pera, 2019). Los beneficios de la inclusión de actividades lúdicas en la educación por medio del juego específicamente en las clases de cultura física ayudan al proceso de enseñanza, un método de prevención en problemas psicológicos como la depresión, el estrés, sedentarismo y problemas de salud mental más graves como el suicidio que pueden ser evitados usando las herramientas que están a nuestro alcance como las actividades lúdicas, en las aulas donde los alumnos pasan gran parte de su tiempo y donde ellos pueden sentirse libres seguros de ser ellos mismos y de crear su propio conocimiento (Gil et al., 2018).

El estado emocional es un sentimiento muy intenso de alegría o de tristeza producido por un hecho, es el sentimiento que se produce frente a determinadas situaciones que aparecen a lo largo de su vida manifestándose por medio de emociones que juegan un papel fundamental en todo el entorno social de cada individuo (Fernández y Jiménez, 2010), las emociones son respuestas que da el individuo y son progresivas, pueden aumentar o disminuir es decir evolucionando dependiendo el estímulo o incidencia que las provocó, pudiendo ser reacciones positivas o negativas dependiendo si uno se siente bien o mal, de duración corta provocando respuestas fisiológicas a nivel del cuerpo como llanto o risa, respuestas neurológicas y cognitivas como la manera de pensar y por ende a la conducta del individuo (Bericat, 2012). Según Piqueras et al. (2006) menciona que las emociones básicas son patrones de conducta con una reacción específica para cada experiencia o sentimiento. Las emociones positivas como, la alegría, el amor y la felicidad tienen determinados beneficios como menciona Fredrickson (2001) estas presentan un motivo esencial debido a que amplían los recursos intelectuales, físicos y sociales del ser humano generando resiliencia frente a las amenazas presentadas a lo largo de la vida de la persona (Barragán y Morales, 2014). Las emociones negativas; miedo, ansiedad, tristeza ira y depresión se conceptualizan como un estado emocional de desagrado (Vaca, 2021).

La actividad lúdica y el estado emocional como lo menciona Goikoetxea et al. (2014) en su estudio acerca de la influencia de los juegos de cooperación-oposición en el ánimo de estudiantes universitarios muestra el grado de relación que existe entre estas dos variables al analizar de manera cuantitativa el efecto de la práctica de juegos en el estado emocional de los estudiantes concluyendo que existe un cambio positivo al realizar actividades lúdicas, de acuerdo al estudio el cambio en los hombres fue menor que en las mujeres, esto se debe a que los hombres tradicionalmente buscan ser altamente competitivos y por ende intensifican el juego mientras que las mujeres buscan una experiencia individual por lo que pueden aprovechar de mejor manera el objetivo del juego. Las emociones están 
presentes en cada ser humano y son parte de nuestro vivir diario y en los estudiantes están presentes en el ámbito educativo, siendo la educación un pilar fundamental para el desarrollo integral de los niños y adolescentes, y el área de educación física un generador de situaciones motrices lúdicas brinda una inmejorable posibilidad para generar experiencias emocionales mediante el uso del juego. La actividad física ayuda en el proceso de formación emocional de los alumnos, por ello es fundamental que en las unidades educativas y en las clases de cultura física se busque la formación emocional de los estudiantes, existen un abanico infinito de actividades lúdicas que se pueden usar para trabajar sus capacidades emocionales desde que ingresan al marco académico, desde los niveles iniciales e ir trabajando junto a ellos nivel a nivel para que luego sean capaces de tener una inteligencia emocional o en palabras más sencillas puedan controlar sus sentimientos apartando los negativos y procurando que los sentimientos positivos predominen (Caballero et al., 2016). Los docentes de educación física deben formarse para tener pleno conocimiento de cada uno de los elementos que compone la práctica física y las emociones que surgen como resultado de dicha práctica para entender su origen significado y poder aplicarlas estrategias pedagógicas que ayuden a la salud mental del estudiante, las emociones que se pueden presentar en un contexto lúdico principalmente emociones positivas como: (alegría, felicidad, amor) en respuesta a una favorable obtención de los objetivos individuales respecto al juego o actividad planteada, por lo cual es necesario que se implementar esta estrategia didáctica en las clases de cultura física para generar experiencias y emocionales positivas en los alumnos que experimentan en las sesiones de la asignatura y como mejorarlas a través del uso de actividades lúdicas que fomente la mejora del estado emocional. En este contexto, el presente documento se lo realizo para definir la asociación entre las actividades lúdicas y el estado emocionales de los estudiantes Unidad Educativa Yanahurco del cantón Mocha.

\section{Metodología}

La presente investigación se realizó con un enfoque cuantitativo ya que utilizo un análisis de tipo estadístico con los datos compilados. De finalidad básica con un diseño no experimental donde no se manipulo las variables de estudio es decir no hubo intervención por parte del investigador. Por su alcance descriptivo al detallar las generalidades de las variables de estudio. De campo con un corte transversal ya que se analizó las variables de estudio en un momento determinado sobre la población con un método del nivel teórico es hipotético-deductivo.

La población de estudio fueron estudiantes de la Unidad Educativa Yanahurco del cantón Mocha, de la que se estableció como muestra 57 niños de octavo, noveno y décimo año del rango de edad de 11 a 13 años. 
Para conocer el nivel de la utilización de la actividad lúdica en los estudiantes, se utilizó una encuesta que permitió identificar la ocupación de esta. Se valoró el nivel del empleo de la actividad lúdica de cada individuo entre baja, modera y alta.

Para medir estados emocionales se utilizó las escalas de Depresión, Ansiedad y Estrés (DASS -21) es un instrumento que mide estados emocionales negativos, consta de 21 ítems, con cuatro alternativas de respuesta en formato tipo Likert, que van desde 0 ("No describe nada de lo que me pasó o sentí en la semana") hasta 3 ("Sí, esto me pasó mucho, o casi siempre"). Los21 ítems de la escala se clasifican en 3 subescalas: Depresión, Ansiedad y Estrés, cada subescala está conformada por 7 ítems con una puntuación máxima de 21 puntos que permite determinar el nivel de severidad de los estados emocionales negativos (ausencia, leve, moderado, severo, extremadamente severo). Con relación a la fiabilidad y validez de la escala varios estudios publicados, consideran al DASS-21 como un instrumento bien estructurado que evalúalos síntomas de depresión, ansiedad y estrés, destacando los índices de consistencia interna que presentaron cada una de las subescalas, así como también de la escala total, con una consistencia interna (Alfa de Cronbach $=0,88$ )

Para el análisis estadístico de los datos y resultados de la presente investigación se lo realizo a través del software estadístico SPSS versión 25 para Windows. En la que realizo de las variables cualitativas frecuencias, porcentajes para profundizar la interpretación descriptiva de las variables.

Para la verificación de la hipótesis de investigación y diferenciación estadística general, se realizó la prueba estadística de normalidad Shapiro-Wilk la cual definió la utilización de pruebas no paramétricas y la prueba estadística de Chi-cuadrado de Pearson para determinar la asociación entre las variables de estudio.

\section{Resultados}

Los resultados obtenidos en la presente investigación fueron realizados a través del software estadístico SPSS de forma cuantitativa. La interpretación de los resultados se encuentra representado en tablas acorde al objetivo planteados en la investigación, los cuales reflejan el nivel de ejercicio físico y el estado de ánimo de los estudiantes. Además, se elaboró una tabla cruzada donde se pudo determinar la asociación entre las variables.

Resultados de la valoración de la ocupación de la actividad lúdica de los estudiantes de octavo, noveno y décimo año de la Unidad Educativa Yanahurco del cantón Mocha.

Aplicando la metodología propuesta para la investigación, a través de la encuesta, se valoró la ocupación de la actividad lúdica entre los niveles bajo que se codifico con el número 1 , moderado con el número 2 y alto con el numero 3 resultados que se evidencian en la tabla 1 y 2. 


\section{Tabla 1}

Evaluación de ocupación de actividades lúdicas

\begin{tabular}{llrrrr}
\hline & Frecuencia & Porcentaje & $\begin{array}{r}\text { Porcentaje } \\
\text { válido }\end{array}$ & $\begin{array}{r}\text { Porcentaje } \\
\text { acumulado }\end{array}$ \\
\hline Válido & Bajo & 32 & 54,2 & 54,2 & 54,2 \\
& Moderado & 14 & 23,7 & 23,7 & 78,0 \\
& Alto & 13 & 22,0 & 22,0 & 100,0 \\
& Total & 59 & 100,0 & 100,0 & \\
\hline
\end{tabular}

Del $100 \%$ de la muestra de estudio el $54.2 \%$ tiene un nivel bajo en ocupación de actividad lúdica, el $23.7 \%$ moderado y solo el $22 \%$ de la muestra presenta un nivel alto en la ocupación de la actividad lúdica. Una vez realizado el análisis de frecuencia encontramos que la mediana y la moda es 1 la cual significa que la ocupación de la actividad lúdica es baja (tabla 2)

\section{Tabla 2}

Mediana y moda de ocupación de la actividad lúdica

\begin{tabular}{lr}
\hline \multicolumn{3}{c}{ Actividad lúdica } \\
\hline Nivel de actividad ludia \\
$\mathrm{N} \quad$ Válido & 59 \\
\multicolumn{2}{c}{ Perdidos } \\
Mediana & 0 \\
Moda & 1,00 \\
\hline
\end{tabular}

Resultados de la identificación del estado emocional de los estudiantes de octavo, noveno y décimo año Unidad Educativa Yanahurco del cantón Mocha.

Aplicando la metodología en base al instrumento DASS21 que permite determinar los estados de ánimo de una población, se evaluaron diferentes coeficientes con base en los estados propuestos cuyos resultados se evidencia en la tabla 3.

\section{Tabla 3}

Análisis estadístico descriptivo de la mediana de los estados emocionales

\begin{tabular}{|c|c|c|c|c|}
\hline \multicolumn{5}{|c|}{ Estados emocionales } \\
\hline & & Nivel de & Nivel de & Nivel de \\
\hline & & depresión & Ansiedad & Estrés \\
\hline \multirow[t]{2}{*}{$\mathrm{N}$} & Válido & 59 & 59 & 59 \\
\hline & Perdidos & 0 & 0 & 0 \\
\hline \multicolumn{2}{|c|}{ Mediana } & 2,00 & 2,00 & 1,00 \\
\hline \multicolumn{2}{|c|}{ Moda } & 1 & 1 & 1 \\
\hline
\end{tabular}


Realizado el análisis estadístico descriptivo encontramos en el estado emocional de la depresión con una md 2 que significa depresión leve, en la ansiedad una md 2 que significa ansiedad leve y en el estrés una md de 1 que corresponde a la ausencia de estrés.

\section{Tabla 4}

Análisis estadístico descriptivo de depresión

\begin{tabular}{llrrrr}
\hline & & Frecuencia & Porcentaje & $\begin{array}{c}\text { Porcentaje } \\
\text { válido }\end{array}$ & $\begin{array}{c}\text { Porcentaje } \\
\text { acumulado }\end{array}$ \\
\hline Válido & & 1 & 1,7 & 1,7 & 1,7 \\
& No depresión & 24 & 40,7 & 40,7 & 42,4 \\
& Depresión leve & 14 & 23,7 & 23,7 & 66,1 \\
& Depresión Moderada & 13 & 22,0 & 22,0 & 88,1 \\
& Depresión severa & 4 & 6,8 & 6,8 & 94,9 \\
Depresión & 3 & 5,1 & 5,1 & 100,0 \\
Extremadamente severa & & & & 100,0 \\
& & & & \\
\hline
\end{tabular}

En la distribución de los estados emocionales negativos de los 59 estudiantes presentaron depresión Leve el 23\%, moderada el 22\%, severa 6,8\% y extremadamente severa el 5,1\%. Se concluye que la depresión leve es la más recurrente en la muestra de estudio.

\section{Tabla 5}

Análisis estadístico descriptivo de Ansiedad

\begin{tabular}{llrrrr}
\hline & Frecuencia & Porcentaje & $\begin{array}{c}\text { Porcentaje } \\
\text { válido }\end{array}$ & $\begin{array}{c}\text { Porcentaje } \\
\text { acumulado }\end{array}$ \\
\hline Válido & No ansiedad & 25 & 42,4 & 42,4 & 42,4 \\
& Ansiedad leve & 8 & 13,6 & 13,6 & 55,9 \\
& Ansiedad Moderada & 15 & 25,4 & 25,4 & 81,4 \\
& Ansiedad severa & 6 & 10,2 & 10,2 & 91,5 \\
Ansiedad & 5 & 8,5 & & 100,0 \\
Extremadamente severa & & & & 100,0 \\
Total & 59 & 100,0 & & \\
\hline
\end{tabular}

En la distribución de los estados emocionales negativos de los 59 estudiantes presentaron Ansiedad Leve el 13,6\%, moderada el 25,4\%, severa 10,2\% y extremadamente severa el $8,5 \%$. Se concluye que la ansiedad moderada es la más recurrente en la muestra de estudio. 


\section{Tabla 6}

Análisis estadístico descriptivo de estrés

\begin{tabular}{llrrrr}
\hline & Frecuencia & Porcentaje & $\begin{array}{c}\text { Porcentaje } \\
\text { válido }\end{array}$ & $\begin{array}{c}\text { Porcentaje } \\
\text { acumulado }\end{array}$ \\
\hline Válido & No estrés & 34 & 57,6 & 57,6 & 57,6 \\
& Estrés leve & 13 & 22,0 & 22,0 & 79,7 \\
& Estrés moderado & 6 & 10,2 & 10,2 & 89,8 \\
Estrés Severo & 5 & 8,5 & 8,5 & 98,3 \\
Estrés extremadamente & 1 & 1,7 & 1,7 & 100,0 \\
Severo & & & & \\
Total & 59 & 100,0 & 100,0 & \\
& & & &
\end{tabular}

En la distribución de los estados emocionales negativos de los 59 estudiantes presentaron estrés leve el $22 \%$, moderada el $10.2 \%$, severa $8.5 \%$ y extremadamente severa el $1.7 \%$. Se concluye que el estrés leve es la más recurrente en la muestra de estudio.

Tabla 7

Análisis estadístico descriptivo del estado emocional

\begin{tabular}{llrrrr}
\hline \multicolumn{5}{c}{ Estado emocional positivos y negativos } \\
\hline \multirow{3}{*}{ Válido } & Frecuencia & Porcentaje & $\begin{array}{c}\text { Porcentaje } \\
\text { válido }\end{array}$ & $\begin{array}{c}\text { Porcentaje } \\
\text { acumulado }\end{array}$ \\
& Positivo & 20 & 33,9 & 33,9 & 33,9 \\
& Negativo & 39 & 66,1 & 66,1 & 100,0 \\
& Total & 59 & 100,0 & 100,0 & \\
\hline
\end{tabular}

De la muestra de estudio de los 59 estudiantes se encontró que el $66.1 \%$ de los estudiantes presentan estados emocionales negativos.

Resultados del uso de juegos lúdicos en relación con el estado emocional de los estudiantes de octavo, noveno y décimo año Unidad Educativa Yanahurco del cantón Mocha.

\section{Tabla 8}

Análisis del uso de juegos lúdicos en relación con el estado emocional

\begin{tabular}{llrrrr}
\hline & & \multicolumn{2}{c}{ Nivel de actividad lúdica } & \\
\cline { 3 - 4 } & & Bajo & Moderado & Alto & Total \\
Estado & Positivo & 3 & 6 & 11 & 20 \\
emocional & Negativo & 25 & 6 & 8 & 39 \\
Total & & 28 & 12 & 19 & 59 \\
\hline
\end{tabular}


Una vez realizado el análisis entre el uso de juegos lúdicos y el estado emocional de los estudiantes se encontró que, de 59 estudiantes, 25 se encontraban con un nivel bajo de uso de actividades lúdicas y un estado emocional negativo.

\section{Verificación de hipótesis}

Aplicando las pruebas estadísticas determinadas para la comparación de significación y asociación entre este tipo de variables cualitativas, se pueden observar los siguientes resultados (tabla 9).

\section{Tabla 9}

Análisis estadístico de significación y asociación entre variables de estudio

\begin{tabular}{lcrr}
\hline \multicolumn{3}{c}{ Pruebas de chi-cuadrado } \\
\hline Valor & df & $\begin{array}{c}\text { Significación } \\
\text { asintótica } \\
\text { (bilateral) }\end{array}$ \\
& & &, 002 \\
Chi-cuadrado de Pearson & $12,988^{\mathrm{a}}$ & 2 &, 001 \\
Razón de verosimilitud & 13,995 & 2 &, 001 \\
Asociación lineal por & 11,742 & 1 & \\
lineal & & & \\
N de casos válidos & 59 & &
\end{tabular}

Una vez aplicada la prueba estadística el Chi-cuadrado de Pearson determino que existe un nivel de asociación entre las variables con un nivel de confiabilidad del 99\% (0.01), con diferenciación asintótica bilateral en un nivel de $\mathrm{P} \leq 0.05$ dando como consecuencia un valor estadístico significativo en el nivel de asociación de las variables de estudio y afirma la aceptación de la hipótesis a que determina:

H1: Las actividades lúdicas se asocia con el estado emocional de los estudiantes.

\section{Discusión}

La presente investigación arroja resultados importantes acerca de las actividades lúdicas y el estado de ánimo, permite establecer la relación del estado de ánimo con el grado de actividad lúdica que presenten los estudiantes, es decir si existe alta presencia de actividades lúdicas los estados de ánimo serán positivos pero si por el contrario se evidencia un nivel bajo de actividad lúdica los estados de ánimo negativos son los que prevalecen, como se determina en el estudio la actividad lúdica es baja en la mayor parte de la población de estudio, esto se refleja en los estados de ánimo que se obtienen en la evaluación los cuales son: presencia de estrés y ansiedad. 
Granados y Cuéllar (2018) menciona que la relación de estado de ánimo y actividad física en todas sus formas se demuestra en varios estudios los cuales llegan a la misma conclusión, la actividad física aumenta la autoconfianza, produce sensaciones de bienestar, mejorar el funcionamiento cognitivo, generando un impacto positivo a nivel; psicológico, calidad de vida y en la mejora del estado emocional en general, también se muestra en la disminución de niveles de ansiedad, depresión y estrés confirmando los resultados obtenidos en este estudio. Otro resultado importante que arroja la investigación es la identificación de estados negativos presentes en estudiantes que se vinculan directamente con la poca práctica de ejercicio físico (juegos) esto a su vez desencadena problemas más graves si no se toma medidas correctivas así lo afirma Bravo et al. (2017) el cual menciona el rol fundamental de las instituciones en cuanto a la enseñanza de la educación emocional porque permite descubrir y desarrollar nuevos tipos de inteligencia, como la emocional mientras se conocen a sí mismos y a sus congéneres que es una función fundamental de los seres humanos, así mismo recomienda a los educadores la importancia de elaborar propuestas metodológicas que incluyan actividades lúdicas que se salgan de lo normal, y que busquen captar la atención de los niños y niñas para que estos se motiven, generen placer y diversión, permitiendo que sus emociones sean expresadas de manera libre y espontánea logrando así un aprendizaje integral.

De igual forma Vilaró (2014) concuerda con los resultados obtenidos y menciona que la escuela es el lugar idóneo para explorar y experimentar con las emociones, a través del juego el cual debe ser estudiado a nivel teórico para descubrir la función del juego con el desarrollo emocional el cual debe ser guiado, observado y evaluado por el docente.

Finalmente se establece que, para tratar problemas asociados con la salud mental, hay una gran variedad de estrategias terapéuticas asociadas con la actividad física, en este caso las actividades lúdicas y su amplia gama de posibilidades se presentan como un aliado para tratar patologías frecuentes hoy en día en estudiantes tales como el estrés, ansiedad y depresión. Pero la actividad física como tal no debe verse únicamente como un proceso correctivo únicamente, si no como un proceso preventivo en donde los problemas son evitados y los beneficios son mayores y en diferentes niveles: a nivel de salud física con la mejora de nuestra apariencia, a nivel de salud psicológica, siendo capaces de controlar y manejar nuestras emociones.

\section{Conclusiones}

- Después de haber realizado la respectiva evaluación y obtener los resultados se llegaron a las siguientes conclusiones.

- Se determinó el bajo empleo de actividades lúdicas en los estudiantes de la Unidad Educativa Yanahurco del cantón Mocha. 
- Los estados emocionales de los estudiantes de la Unidad Educativa Yanahurco del cantón Mocha donde presentaron mayor incidencia fueron depresión leve, ansiedad leve y la ausencia de estrés.

- Se comprobó que existe una asociación estadísticamente significativa entre las variables de estudio actividades lúdicas y estados emocionales de los estudiantes.

- El realizar actividades lúdicas que actúan como factor protector para evitar desarrollar estados emocionales negativos en los estudiantes.

\section{Referencias bibliográficas}

Barragán, E. A., \& Morales, M. C. (2014). Psicología de las emociones positivas: generalidades y beneficios. Redalyc.org, 19(1), 103-118. Obtenido de https://www.redalyc.org/pdf/292/29232614006.pdf

Benítez, L. M. T., \& Gamarro, F. H. (2010). La Expresión corporal en Educación Física: Propuesta curricular desde la administración educativa. Wanceulen SL

Bericat, E. (2012). Emociones. Universidad de Sevilla. Arrangement of Sociopedia.isa. doi:10.1177/205684601261

Bravo Lanzaque, Silvia de la Caridad, Pérez Clemente, Yamilet, González Barreto, Marilyn, Campos Maura, Eraida, \& Díaz Díaz, Oliday. (2021). Los juegos didácticos en la clase de consolidación de Matemática en la secundaria básica cubana. Dilemas contemporáneos: educación, política y valores, 8(2), 00017. Epub 21 de abril de 2021.https://doi.org/10.46377/dilemas.v8i2.2527

Caballero García, M., Roque, A., \& Yuste, L. (2016). Intensidad emocional en la clase de educación física en función de la victoria: juegos de cooperación-oposición. Revista Electrónica Interuniversitaria de Formación del Profesarado, 123-133.

Fernández, A. E., \& Jiménez, S. M. (2010). Psicología de la emoción. En E. G.-A. Sánchez, Psicología de la emoción (págs. 17 - 74). Universitaria Ramón Areces y UNED. Obtenido de https://www.cerasa.es/media/areces/files/book-attachment2986.pdf

Fredrickson, B. L. (2001). The role of positive emotions in positive psychology: the broaden-and-build theory of positive emotions. American psychologist, 56(3), 218.

Gil, F., Romance García, Á. R., \& Rodríguez, N. (2018). Juego y actividad física como indicadores de calidad en Educación infantil. Federación Española de Asociaciones de Docentes de Educación Física, 252-257. 
Goikoetxea, A., Santos Gorostiaga, R. M., Arruabarrena, O., Otegi, Joseba, \& Liebaert, C. (2014). Efectos de los juegos de cooperación-oposición en el ánimo de los universitarios. Federación Española de Asociaciones de Docentes de Educación Física, 58-62.

Gómez-Mármol, A., Sánchez-Alcaraz Martínez, B. J., \& Bazaco Belmonte, M. J. (2017). La Educación Física como asignatura lúdica en Educación Secundaria: sedentarismo y práctica de actividades físico-deportivas extraescolares. Sportis, 3(3), 605-620.

Granados, S. H. B., \& Cuéllar, Á. M. U. (2018). Influencia del deporte y la actividad física en el estado de salud físico y mental: una revisión bibliográfica. Katharsis: Revista de Ciencias Sociales, (25), 141-160.

Osorio Angarita, M. A., Suárez Parra, A., \& Uribe Sandoval, C. C. (2013). Revisión de alternativas propuestas para mejorar el aprendizaje de la Probabilidad. Revista Virtual Universidad Católica Del Norte, 1(38), 127-142. Recuperado a partir de https://revistavirtual.ucn.edu.co/index.php/RevistaUCN/article/view/409

Palacios, F. J. P., \& de Dios Jiménez, J. (2002). Las ilustraciones en la enseñanzaaprendizaje de las ciencias. Análisis de libros de texto. Enseñanza de las ciencias: revista de investigación y experiencias didácticas, 369-386.

Pera, C. A. (2019). Importancia de la Actividad Física. Revista Médico-Científica de la Secretaría de Salud Jalisco, 122-127.

Piqueras, J., Ramos, R., \& García, L. (2006). Ansiedad, Depresión y salud en L.A. Oblitas (ed.), Psicología de la salud y enfermedades crónicas. Bogotá: Psicomo editores.

Romero, L., Escorihuela, Z., \& de Balazs, A. C. R. (2009). La actividad lúdica como estrategia pedagógica en educación inicial. Lecturas: Educación física y deportes, (131), 46-46.

Mocha-Bonilla, J. A., Barquin, C., \& Castro, W. (2018). Efectos de un programa de juegos recreativos en la definición de la lateralidad. Revista ESPACIOS, 39(23).

Vaca, D., \& Mayorga, D. (2021). Estados emocionales y su relación con el insomnio en el personal de salud durante pandemia. Enfermería Investiga, 6(1), 20-26.

Vilaró-Tió, M. (2014). El desarrollo emocional a través del juego: Propuesta de intervención para alumnos del segundo ciclo de Educación Infantil (Bachelor's thesis).

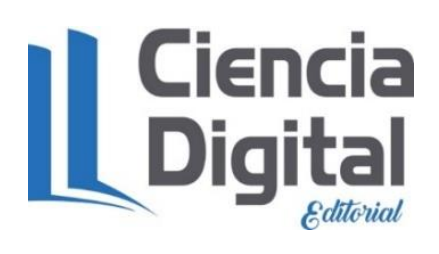


El artículo que se publica es de exclusiva responsabilidad de los autores y no necesariamente reflejan el pensamiento de la Revista Conciencia Digital.

\section{Ciencia \\ LDigital}

El artículo queda en propiedad de la revista y, por tanto, su publicación parcial y/o total en otro medio tiene que ser autorizado por el director de la Revista Conciencia Digital.
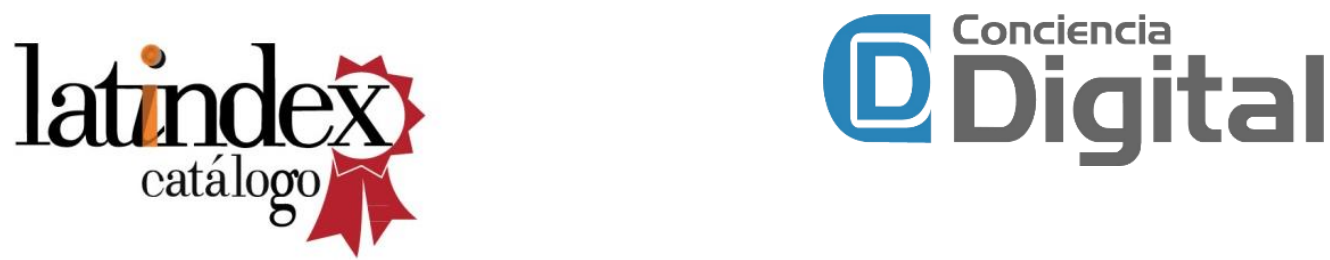

Indexaciones

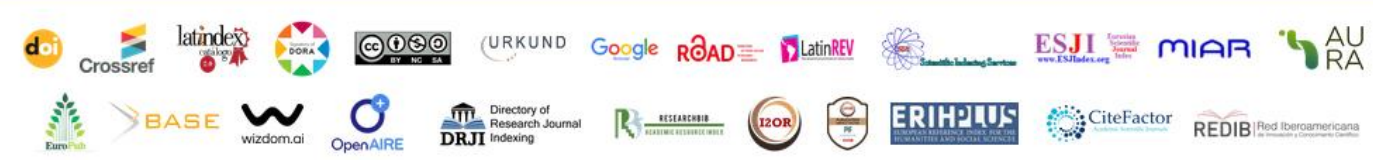

